\title{
Reproductive Performance of Female Nile Tilapia Oreochromis niloticus L. In South Kivu (DR Congo): Comparison of Two Strains Differing In Origin and History of Domestication
}

(Performances de reproduction des femelles du tilapia du Nil Oreochromis niloticus L. au Sud-Kivu (RD Congo) : comparaison de deux souches différant par leur origine et leur histoire de domestication)

Nihoreye, F.J..$^{*}$, Nyongombe, U.N. ${ }^{2}$, Alunga, L.G. ${ }^{3,4}$, Akonkwa, B.D. ${ }^{5}$, Isumbisho, M.P. ${ }^{6}$ et Okitayela, O.F. ${ }^{7}$

${ }^{1}$ Département de Biologie, Institut Supérieur Pédagogique de Bukavu. BP 854 Bukavu, RD Congo

${ }^{2}$ Faculté des Sciences Agronomiques, Département de Zootechnie, Université Pédagogique Nationale. BP 8815 Kinshasa, RD Congo

${ }^{3}$ Centre de Recherches Universitaires du Kivu, Institut Supérieur Pédagogique de Bukavu. BP 854 Bukavu, RD Congo

${ }^{4}$ Unité d'Enseignement et de Recherche en Hydrobiologie Appliquée, Institut Supérieur Pédagogique de Bukavu. BP 854 Bukavu, RD Congo

${ }^{5}$ Faculté des Sciences, Département de Biologie, Université Officielle de Bukavu. BP 570 Bukavu, RD Congo

${ }^{6}$ Unité de Recherche en Gestion des Ecosystèmes Humides et Aquatiques, Institut Supérieur Pédagogique de la Gombe. BP 3580 Kinshasa, RD Congo

${ }^{7}$ Département de Zootechnie, Faculté des Sciences Agronomiques, Université de Kinshasa. BP 117 Kinshasa XI, RD Congo

\begin{abstract}
This study was conducted to evaluate the reproductive performance of females of Oreochromis niloticus originating from a strain of distant origin and extensively exploited in fish farming (Nyakabera strain) and a local strain recently removed from the natural environment (Lac Kivu strain). The experiment was carried out in triplicate with fry of 4.9 to $6.3 \mathrm{~g}$ placed in earthen ponds for a period of 6 months, at the Nyakabera fish station in South Kivu, Democratic Republic of Congo. The comparison of the two strains focused on the size of the first sexual maturity, gonado-somatic ratio, fecundity, egg diameter and condition factor. The results showed that under similar managed rearing conditions, the Nyakabera strain reaches sexual maturity earlier and is characterized by a higher gonado-somatic ratio and higher relative fertility, but a worse condition compared to the Lac Kivu strain. These differences, which indicate a better reproductive success of the Nyakabera strain, are related to the origin and the history of domestication, which are just as different from these two strains.
\end{abstract}

Key words: Fertility, Fish Farming, Gonado-Somatic Ratio, Lake Kivu, Size Of First Sexual Maturity

\section{Introduction}

Le tilapia du Nil Oreochromis niloticus est une espèce de poisson dont l'importance économique et aquacole à l'échelle mondiale n'est plus à démontrer.

En système d'élevage en sexes mélangés en étang, encore très courant en contexte paysan, O. niloticus présente certaines caractéristiques reproductives, telles que la prolificité, la maturation précoce, la fréquence des pontes, qui sont jugées intéressantes ou au contraire indésirables selon que les performances visées se rapportent à la production d'alevins ou au grossissement. C'est ainsi que, selon Mair et al. (2004), une maturité sexuelle tardive et, dans une moindre mesure, une fécondité réduite sont considérées comme des 
traits avantageux pour les producteurs des poissons marchands mais indésirables pour les producteurs d'alevins.

Dans le même sens, Smitherman et al. (1988) notent que les différences entre souches, la consanguinité et l'hybridation peuvent affecter les performances de reproduction chez les poissons d'élevage. D'importantes différences entre souches ont été mises en évidence à ce sujet par exemple pour le poisson-chat américain (Dunham et Smitherman, 1984) et la truite arc-en-ciel (Kincaid, 1981).

Par ailleurs, les tilapias sont reconnus pour leur variabilité par rapport aux traits reproductifs (Little, 1989; Mair et al., 2004 ; Kamel, 2011), ceci à l'intérieur et entre les souches (Rana et Macintosh, 1988 ; Smitherman et al., 1988; Bolivar et al., 1993; Eguia, 1996). D’ après de Graaf et al. (1999), le potentiel de reproduction d'O. niloticus est influencé notamment par l'environnement et les différences entre les souches.

L'intérêt porté à l'élevage d'O. niloticus à travers le monde a comme conséquence l'existence d'une diversité de souches en circulation. En 2012, FishBase a inventorié 30 souches d'O. niloticus avec pour chacune son origine et son histoire de domestication

(http://www.fishbase.org/physiology/strainlist.php?ID=2\&SortBy=StrainCode). Osure et Phelps (2006) en signalent six sources initiales sur lesquelles est basée la majorité de la production mondiale, à savoir Côte d'Ivoire, Ghana, Egypte-Manzala, Egypte-Ismailia, Nile-Soudan et Lac George-Ouganda. Il a été décelé une différenciation morphologique et/ou génétique entre la plupart de ces souches (Gourène et Teugels, 1993; Macaranas et al., 1995 ; Vreven et al., 1998).

Selon Duponchelle et al. (1998), les souches d'O. niloticus peuvent aussi montrer d'importantes différences notamment dans leurs traits reproductifs. Certaines études ont mis en évidence l'existence d'une relation entre les performances reproductives et l'origine et/ou l'histoire de domestication des populations de poissons en général (Stearns, 1983) et d'O. niloticus en particulier (Duponchelle et al., 1998; Mair et al, 2004 ; Osure et Phelps, 2006). D'après Stearns (1983), les différences observées dans l'origine et/ou l'histoire de vie (des populations de poissons) ont généralement des composantes environnementales et génétiques.

O. niloticus se trouve dans la région du Sud-Kivu (en RD Congo) à l'état naturel, dans les lacs et certains cours d'eau, et en situation d'élevage dans les étangs piscicoles où il constitue la principale espèce utilisée. Une récente étude comparative portant sur l'aspect morphologique (Nihoreye et al., sous presse) a montré une nette démarcation d'une souche domestique allochtone largement répandue dans cette région (« souche Nyakabera ») par rapport à deux populations naturelles autochtones. L'une d'elles, dite « souche lac Kivu », est en essai d'acclimatation en station de pisciculture depuis 2014.

Dans le cadre d'un aperçu sur les aspects de la reproduction des poissons du lac Kivu, Akonkwa et al. (2016) ont évalué quelques traits reproductifs d'O. niloticus. De toute évidence, aucune recherche n'a été entreprise jusque-là sur la biologie de la reproduction d'O. niloticus en contexte d'élevage dans la région du Sud-Kivu, alors que, comme le soulignent Albaret (1982) et Almeida et al. (2013), la connaissance du potentiel de reproduction d'une espèce de poisson peut permettre la sélection des souches intéressantes en matière d'élevage.

Ainsi, la présente étude se propose de déterminer et de comparer la taille de première maturité sexuelle, le rapport gonado-somatique, le facteur de condition et la fécondité des deux souches du tilapia du Nil Oreochromis niloticus présentes au Sud-Kivu, différant par leur origine et leur histoire de domestication. Ceci en vue d'identifier la souche qui manifesterait le meilleur succès reproductif dans le contexte local d'élevage.

\section{Matériel et méthodes}




\subsection{Site d'étude}

Cette étude a été conduite de mars à septembre 2017 à la station piscicole étatique de Nyakabera, située en périphérie de la ville de Bukavu, en Province du Sud-Kivu, République Démocratique du Congo. La station piscicole de Nyakabera est située à $02^{\circ} 28^{\prime}$ de latitude Sud, $28^{\circ} 49^{\prime}$ de longitude Est et à $1553 \mathrm{~m}$ d'altitude. Le site compte actuellement 37 étangs de dérivation en terre (totalisant 98,7 ares) dédiés essentiellement à la production des alevins.

\subsection{Souches de poisons utilisées}

La présente étude a porté sur deux souches du tilapia du Nil Oreochromis niloticus, respectivement nommées « Nyakabera » et « lac Kivu », élevées à la station piscicole de Nyakabera.

La souche d'O. niloticus dite « lac Kivu» existe à la station piscicole de Nyakabera depuis avril 2014. Elle a été introduite à Nyakabera depuis le lac Kivu, bassin de Bukavu, dans le cadre d'un essai d'acclimatation. O. niloticus est la seule espèce autochtone sur les 4 espèces de tilapia signalées au lac Kivu (Snoeks et al., 1997). Les poissons de cette souche ayant fait l'objet de ce travail appartenaient à la cinquième génération depuis son introduction à la station piscicole de Nyakabera.

La souche domestique d'O. niloticus dite « Nyakabera », utilisée dans cette étude, a été introduite en 2005 à Nyakabera depuis Rwasave, une station piscicole de l'Université Nationale du Rwanda à Butare. Elle est à l'origine un mélange de souches égyptienne et ivoirienne, elles-mêmes amenées à Rwasave depuis une station de pisciculture de l'Université d'Auburn (USA) entre 1984 et 1985 (Karen Veverica, comm. pers., 2009). Il s'agirait de la souche « Rwanda-USA 1984 », selon FishBase, dont le stock fondateur était de 22 femelles et 18 mâles (http://www.fishbase.org/physiology/strainlist.php?ID=2\&SortBy=StrainCode). Depuis son arrivée à la station de Nyakabera, cette souche est disséminée dans plusieurs contrées des provinces de l'Est de la RD Congo à travers des programmes d'assistance aux paysans pisciculteurs implémentés notamment par les organisations humanitaires (Nihoreye et al., 2009).

\subsection{Protocole expérimental}

L'expérimentation a été conduite en triplicata dans six étangs en terre de 2 ares avec une profondeur moyenne de $1 \mathrm{~m}$ chacun. Les étangs étaient alimentés en eau à travers un canal dérivé à partir de la rivière Nyakabera située à environ $200 \mathrm{~m}$. Pour limiter l'invasion des étangs expérimentaux par des poissons étrangers indésirables, un treillis métallique à mailles fines a été fixé à chaque tuyau devant servir d'alimentation en eau de l'étang.

La mise en charge des étangs expérimentaux a été réalisée à un taux uniforme de 1 poisson $/ \mathrm{m}^{2}$. Les alevins utilisés avaient les poids moyens ( \pm écart-type) initiaux de 4,9 $\pm 0,8 \mathrm{~g}$ et 6,3 $\pm 0,6 \mathrm{~g}$, respectivement pour la souche du lac Kivu et la souche Nyakabera. Les deux lots d'alevins ont été jugés utilisables pour l'expérimentation après avoir vérifié que leur différence par rapport au poids n'était pas statistiquement significative ( $\mathrm{p}>0,05$; test $\mathrm{U}$ de Mann-Whitney).

Avant l'empoissonnement, les étangs ont été drainés et chaulés avec de la chaux agricole à raison de 0,25 $\mathrm{kg} / \mathrm{m}^{2}$ (Engle et Neira, 2005). Cette opération a permis de réguler le $\mathrm{pH}$ de l'eau et éliminer les parasites tels que les sangsues et les larves des grenouilles. La fertilisation était partout réalisée avec des feuilles de Tithonia diversifolia placées dans une compostière de $4 \mathrm{~m}^{2}$ aménagée dans un coin de l'étang. Les compostières étaient rechargées une fois par semaine. Les poissons étaient nourris avec un aliment constitué d'un mélange de drèche de brasserie $(50 \%)$, de tourteau palmiste $(30 \%)$ et de farine de fretin $(20 \%)$. La distribution de l'aliment se faisait deux fois par jour, le matin entre 9 heures et 10 heures et le soir entre 15 heures et 16 heures. Le taux journalier de nourrissage appliqué était de 5\% de la biomasse estimée des 
poissons (à partir d'un échantillonnage réalisé mensuellement). L'expérimentation s'était étendue sur une période de 6 mois.

\subsection{Détermination des paramètres de reproduction}

Après 6 mois d'élevage, les étangs ont été complètement vidés afin de capturer tous les poissons. Un échantillon constitué de 200 spécimens de différentes tailles par souche a été prélevé afin d'en déterminer les paramètres de reproduction. Par la suite, ces poissons ont été pesés au gramme près puis mesurés au millimètre près avant dissection et prélèvement des gonades. Ces dernières ont été également pesées (à 0,01 $\mathrm{g}$ près) et seules celles contenant des ovocytes mûrs prêts pour la ponte ont été conservées dans du formol $5 \%$ pour des analyses ultérieures.

L'analyse des paramètres de reproduction, notamment l'état de maturité sexuelle, le rapport gonadosomatique (RGS), le facteur de condition (K), la fécondité et la taille des ovocytes, a été réalisée par la suite uniquement pour les individus femelles. L'échantillon de poissons examinés comprenait, pour la souche Nyakabera, 108 femelles d'une taille (longueur totale) comprise entre $11,1 \mathrm{~cm}$ et $18,1 \mathrm{~cm}$ et, pour la souche Lac Kivu, 138 femelles d'une taille comprise entre 9,5 cm et 22,2 cm.

La méthode utilisée pour chaque paramètre se résume comme suit :

Détermination des stades de maturité des gonades

Les stades de maturité des gonades ont été déterminés par un examen macroscopique basé sur l'échelle à 6 stades (Tableau 1) adaptée de Fermon (1996).

Tableau 1 : Description des stades de maturité des femelles d'O. niloticus telle qu'adaptée de Fermon (1996)

\begin{tabular}{|l|l|}
\hline Stade 0: & Gonades invisibles \\
\hline Stade 1 : & Sexe incertain mais gonades présentes \\
\hline Stade 2 $=6:$ & $\begin{array}{l}\text { Ovaires vides, differenciés. Présence d'ovules très petits, jaunâtres, peu } \\
\text { nombreux }\end{array}$ \\
\hline Stade 3: & Ovules petits mais remplissant entièrement les ovaires \\
\hline Stade 4: & Ovaires comportant des ovules de diamètre différent et des inclusions \\
\hline Stade 5: & $\begin{array}{l}\text { Ovaires remplis uniquement d'ovules de même diamètre non expulsables par } \\
\text { pression manuelle }\end{array}$ \\
\hline Stade 5+: & Ovules expulsables par pression manuelle \\
\hline
\end{tabular}

Estimation de la taille de première maturité sexuelle

En accord avec Legendre et Ecoutin (1989), la taille de première maturité sexuelle $\left(\mathrm{L}_{50}\right)$ est définie ici comme étant la longueur totale à laquelle $50 \%$ des poissons sont à un stade avancé (stades 5 ou $5+$ de l'échelle de maturité utilisée) de leur premier cycle sexuel. Pour estimer la taille de première maturité sexuelle, les individus ont été regroupés par classes de taille $(\mathrm{Lt}) \mathrm{de} 1 \mathrm{~cm}$. Dans chaque classe de taille, le pourcentage des individus matures a été calculé. Une fonction logistique d'une régression non linéaire reliant les pourcentages des individus matures et les tailles $(\mathrm{Lt})$ des poissons a été utilisée. La courbe de cette fonction de forme sigmoïde permet de déterminer la $\mathrm{L}_{50}$. L'équation de cette fonction s'écrit comme suit (Berté et al., 2008) :

$\mathrm{P}=\mathrm{e}^{(\mathrm{a}+\mathrm{bLt})} / 1+\mathrm{e}^{(\mathrm{a}+\mathrm{bLt})}$, avec $\mathrm{P}$ : pourcentage des individus matures; Lt: longueur totale; a et $\mathrm{b}$ : constantes. La taille de première maturité sexuelle $\left(L_{50}\right)$ était ainsi dérivée de la relation entre $a$ et $b: L_{50}=-a / b$. 
Le rapport gonado-somatique (RGS) est une mesure qui décrit l'état de maturité d'un poisson en exprimant le poids des gonades en pourcentage du poids corporel. Normalement, la valeur du RGS croît lorsque le développement des gonades approche la maturité ; la valeur commence à décroître lorsque le poisson commence à pondre (Shoko et al., 2015). Le RGS a été calculé par la formule (De Vlaming et al., 1982):

$(\mathrm{PGo} \times 100) / \mathrm{Pt} ;$ avec $\mathrm{PGo}=$ poids des gonades (en gramme) $\mathrm{Pt}=$ poids total du corps (en gramme)

Le facteur de condition (K) est utilisé pour comparer la condition («embonpoint ») et le bien-être d'un poisson et, selon Bagenal (1978), les poissons plus lourds à une longueur donnée sont en meilleure condition. Le facteur de condition a été calculé comme suit (Pauly, 1993) : K $=(\mathrm{Px} 100) / \mathrm{L}^{3}$, où $\mathrm{P}$ et $\mathrm{L}$ sont le poids individuel du poisson (en gramme) et la longueur totale (en centimètre), respectivement.

Détermination de la fécondité et du diamètre des ovocytes

La fécondité est estimée à partir des gonades en stades de maturation finale par le comptage des ovocytes ayant le plus grand diamètre (Duponchelle et al., 2000). La fécondité absolue est le nombre total d'ovocytes mûrs avant la prochaine période de ponte. La fécondité relative est le nombre total d'ovocytes mûrs par gramme de poids corporel de la femelle (Bagenal, 1978).

Pour obtenir des échantillons représentatifs de toute la gonade, de petites portions étaient prélevées sur les régions postérieure, médiane et antérieure de deux lobes de l'ovaire. Ces échantillons étaient pesés et les ovocytes mûrs comptés. Le nombre total d'ovocytes mûrs dans l'ovaire était estimé en multipliant le nombre d'ovocytes mûrs dans l'échantillon par le rapport du poids de l'ovaire au poids moyen calculé sur les échantillons (Hunter et al., 1992 ; Shalloof et Salama, 2008).

Le diamètre ovocytaire a été estimé à partir des mensurations faites sur 30 ovocytes pris au hasard sur les gonades d'une femelle au stade de maturation $5 / 5+$. Le diamètre ovocytaire a été déterminé en calculant la moyenne arithmétique des mensurations relevées sur le grand diamètre de l'ensemble des ovocytes mesurés (Legendre et Ecoutin, 1996).

\subsection{Analyses statistiques des données}

Le test non paramétrique U de Mann-Withney a permis de comparer les deux souches d'O. niloticus étudiées par rapport à la fécondité, au rapport gonado-somatique, au diamètre ovocytaire et au facteur de condition. L'analyse de la régression linéaire a été utilisée pour tester la relation entre la fécondité et le poids total, la fécondité et le poids des gonades et la fécondité et le diamètre ovocytaire. Toutes les analyses statistiques ont été réalisées grâce au logiciel PAST, version 3.18 (Hammer et al., 2001). Le logiciel Microsoft Excel 2010 a servi pour les différents calculs et les graphiques. La signification des différences a été considérée au seuil de $5 \%$.

\section{Résultats}

\subsection{Taille de première maturité sexuelle}

Les femelles des souches d'O. niloticus Nyakabera et Lac Kivu atteignent la $\mathrm{L}_{50}$ à une longueur totale de $10,6 \mathrm{~cm}$ et 13,5 cm, respectivement (Figure 1). A ces tailles ainsi estimées correspondent respectivement les poids de 12,6 g et 35,2 g, d'après un calcul de la régression linéaire. 


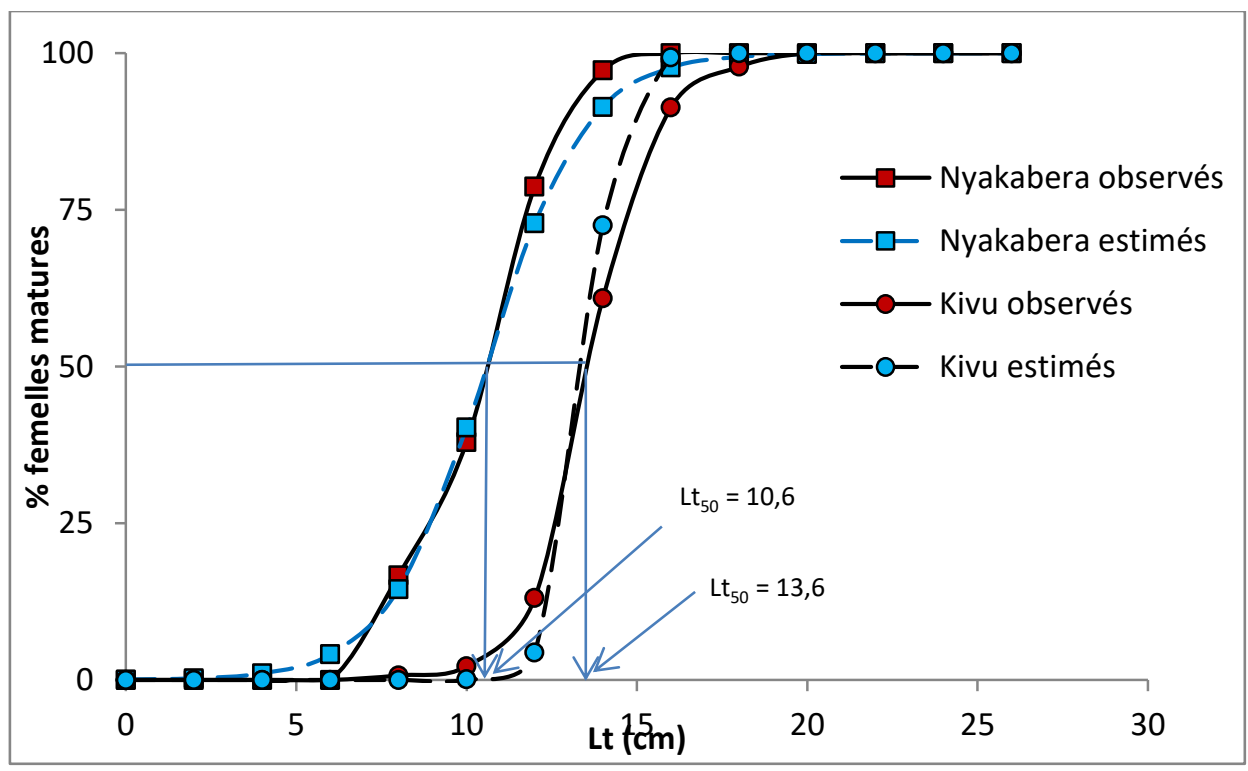

Figure 1 : Estimation de la taille de première maturité sexuelle des femelles des souches Nyakabera et Lac Kivu d'O. niloticus

\subsection{Rapport gonado-somatique et facteur de condition}

Le rapport gonado-somatique varie de 0,35 à 5,03\%, avec une moyenne de 2,50 $\pm 0,09 \%$ chez la souche du

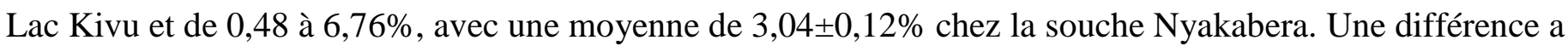
été observée par rapport au RGS entre les femelles de deux souches $(p=0,005)$, la souche Nyakabera ayant un RGS moyen plus élevé que la souche Lac Kivu (Tableau 2).

Tableau 2 : Rapport gonado-somatique (RGS; \% du poids corporel) et facteur de condition (K) des femelles des souches Lac Kivu et Nyakabera d'O. niloticus

\begin{tabular}{|l|l|l|l|l|l|}
\hline \multicolumn{3}{|l|}{ Lac Kivu } & Nyakabera & \\
\hline Paramètre & Min-Max & Moy & Min-Max & Moy & $P$ \\
\hline RGS & $0,35-5,03$ & $2,50 \pm 0,09$ & $1,34-3,91$ & $3,04 \pm 0,12$ & 0,005 \\
\hline K & $0,48-6,76$ & $1,70 \pm 0,03$ & $0,21-3,28$ & $1,61 \pm 0,02$ & $<0,001$ \\
\hline
\end{tabular}

La lecture du tableau ci-dessus montre que les deux souches sont également différentes $(\mathrm{p}<0,001)$ par rapport au facteur K. La souche Lac Kivu se caractérise par une condition relativement meilleure (valeurs de $\mathrm{K}$ variant de 1,34 à 3,91) que la souche Nyakabera (valeurs de $\mathrm{K}$ variant de 0,21 à 3,28).

\subsection{Fécondité et diamètre ovocytaire}

La fécondité et le diamètre ovocytaire ont été analysés, pour chacune de deux souches, sur un échantillon aléatoire de 50 femelles aux stades de maturité sexuelle 5 et $5+$. Les données relatives à la fécondité et la taille des ovocytes sont présentées dans le tableau 3.

Tableau 3 : Fécondité et diamètre ovocytaire des femelles des souches Lac Kivu et Nyakabera d'O. niloticus

\begin{tabular}{|l|l|l|l|l|l|}
\hline & Lac Kivu & & Nyakabera & & \\
\hline Paramètres & Min-Max & Moy & Min-Max & Moy & P \\
\hline $\begin{array}{l}\text { Fécondité } \\
\text { absolue }\end{array}$ & $279-1570$ & $675 \pm 39,8$ & $216-1504$ & $508 \pm 31,9$ & $<0,001$ \\
\hline Fécondité relative & $3,17-15,7$ & $7,70 \pm 0,38$ & $5,49-17,2$ & $12,2 \pm 0,33$ & $<0,001$ \\
\hline Diamètre & $0,89-2,15$ & $1,59 \pm 0,05$ & $0,95-2,00$ & $1,63 \pm 0,05$ & 0,40 \\
\hline
\end{tabular}




\begin{tabular}{|l|l|l|l|l|l|}
\hline ovocytaire $(\mathrm{mm})$ & & & & & \\
\hline $\begin{array}{l}\text { Longueur totale } \\
(\mathrm{cm})\end{array}$ & $14,6-22,2$ & $16,6 \pm 0,16$ & $11,6-18,1$ & $13,6 \pm 0,14$ & $<0,001$ \\
\hline
\end{tabular}

Le nombre d'ovocytes mûrs par femelle (fécondité absolue) d'O. niloticus des souches Lac Kivu et Nyakabera varie respectivement de 279 à 1570 et 216 à 1504. Ces valeurs correspondent à des longueurs totales de 14,6-22,2 et 11,6-18,1 cm, respectivement. Ce nombre, tout comme la longueur totale des poissons, est en moyenne plus élevé $(\mathrm{p}<0,001)$ dans la souche Lac Kivu. La tendance est inversée pour la fécondité relative pour laquelle il a été observé 7,70 \pm 0,38 ovocytes/g de femelle chez Lac Kivu contre 12,2 $\pm 0,33$ ovocytes/g de femelle chez Nyakabera.

Le diamètre ovocytaire varie de 0,89 à $2,15 \mathrm{~mm}$, avec une moyenne de 1,59 $\pm 0,05 \mathrm{~mm}$ et de 0,95 à 2,00 $\mathrm{mm}$, avec une moyenne de 1,63 $\pm 0,05 \mathrm{~mm}$ respectivement pour les femelles provenant du Lac Kivu et de Nyakabera. Aucune différence n'a été détectée entre les deux souches par rapport à ce paramètre $(p=0,40)$.

Par ailleurs, au niveau de deux souches, on a observé que la fécondité absolue est positivement corrélée avec la longueur totale et le poids total du corps et avec le poids des ovaires mais ne manifeste pas de corrélation avec le diamètre des ovocytes (Figures 2, 3, 4 et 5). Par rapport aux trois premiers paramètres précités (longueur totale, poids total et poids des ovaires), la corrélation avec la fécondité absolue est chaque fois plus forte pour la souche Nyakabera que pour la souche lac Kivu (voir valeurs respectives du coefficient de corrélation $r$ et de $p$ ).
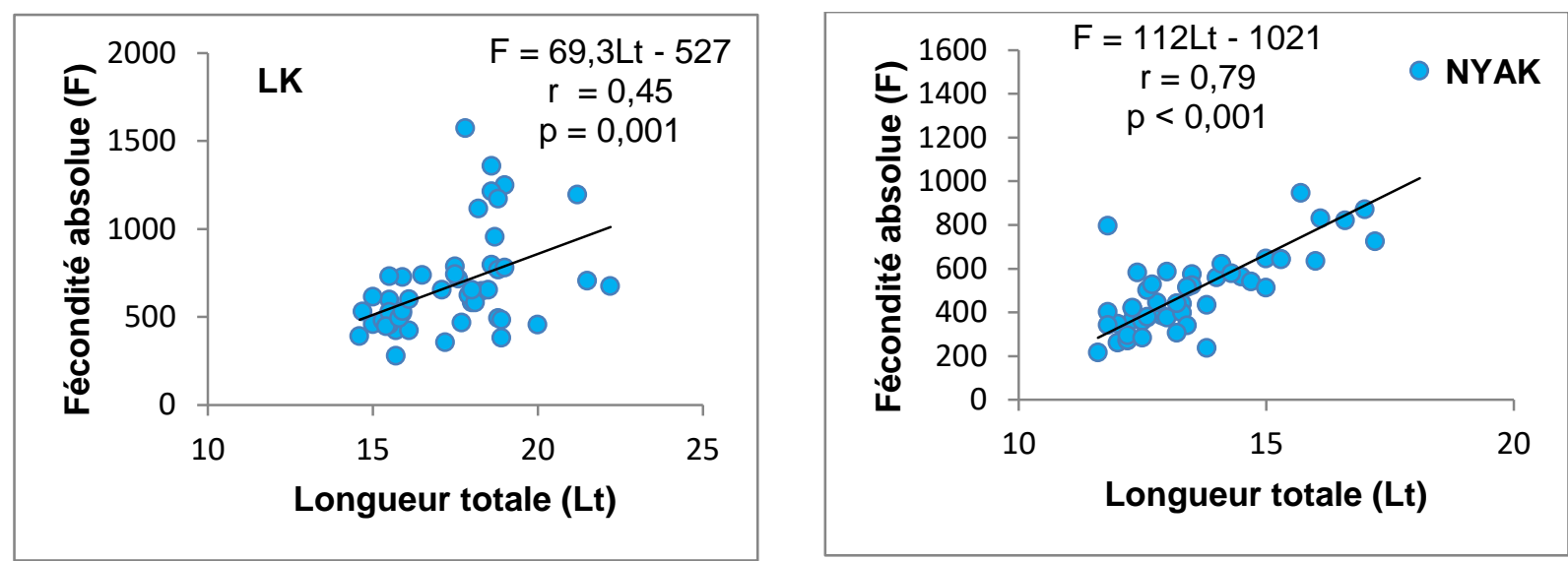

Figure 2 : Relation entre la fécondité absolue et la longueur totale chez les souches lac Kivu (LK) et Nyakabera (NYAK) d'O. niloticus
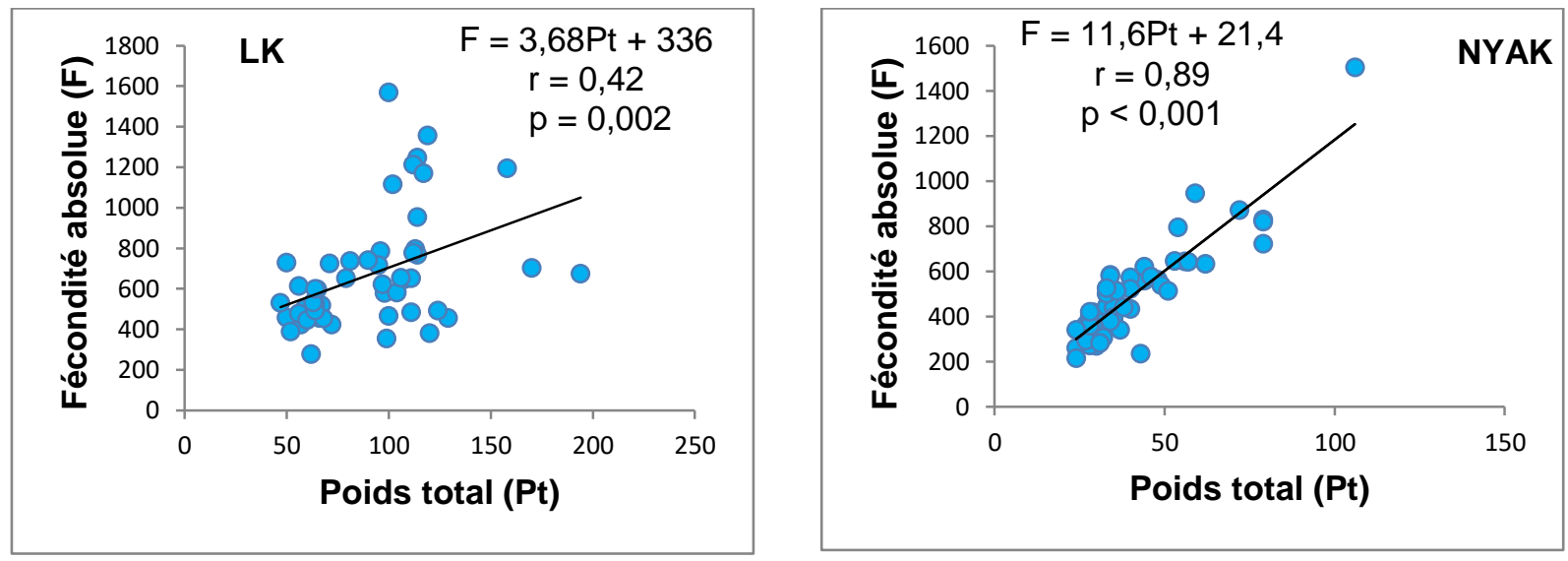
Figure 3: Relation entre la fécondité absolue et le poids total chez les souches lac Kivu (LK) et Nyakabera (NYAK) d'O. niloticus
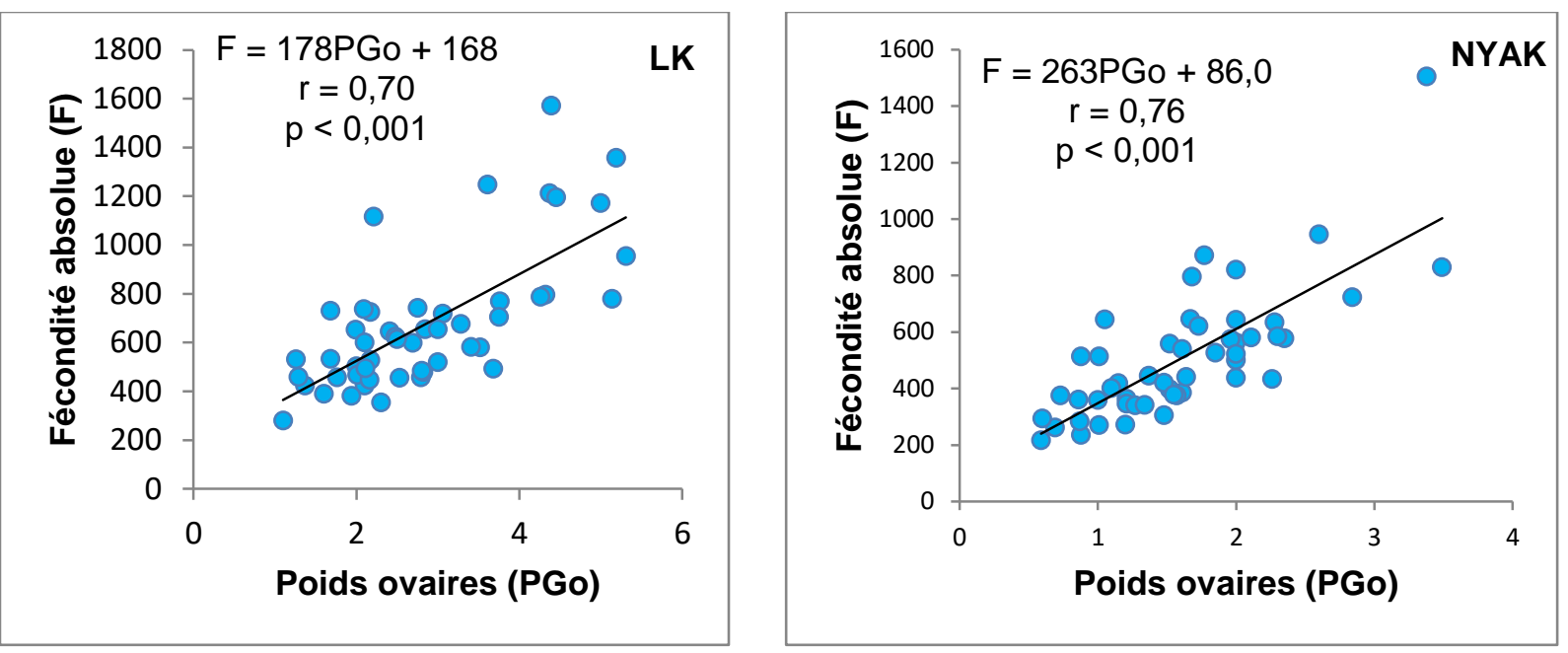

Figure 4 : Relation entre la fécondité absolue et le poids des ovaires chez les souches lac Kivu (LK) et Nyakabera (NYAK) d'O. niloticus
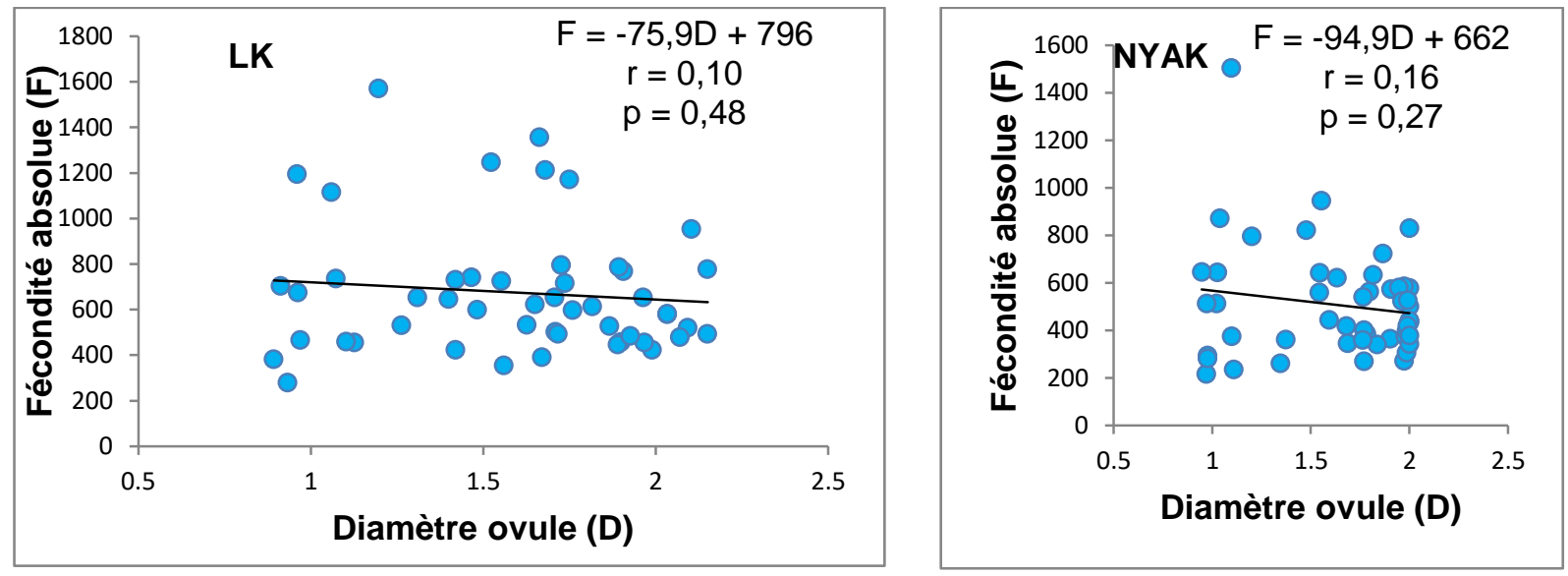

Figure 5 : Relation entre la fécondité absolue et le diamètre ovocytaire chez les souches lac Kivu (LK) et Nyakabera (NYAK) d'O. niloticus

\section{Discussion}

La présente étude a été conduite dans le but de déterminer et de comparer la taille de première maturité sexuelle, le rapport gonado-somatique, le facteur de condition et la fécondité des femelles de deux souches du tilapia du Nil Oreochromis niloticus trouvées actuellement dans la région du Sud-Kivu, en RD Congo. L'une de ces souches (Nyakabera), d'origine allochtone, a une ancienne histoire dans la domestication tandis que l'autre, prise localement en milieu naturel (le lac Kivu) il y a moins de cinq ans, était à sa cinquième génération en conditions d'élevage. Ces deux souches présentent en outre des différences morphologiques (Nihoreye et al., sous presse). Les résultats de la présente étude ont montré que les deux souches étudiées, soumises à des conditions d'élevage similaires, diffèrent pour quatre sur les cinq paramètres de reproduction examinés. Ceci va dans le sens des observations antérieures (Duponchelle et al., 1998) selon lesquelles les traits reproductifs chez $O$. niloticus sont influencés notamment par les différences entre les souches ; y compris par rapport à l'origine et à l'histoire de domestication de celles-ci (Mair et al., 2004 ; Osure et Phelps, 2006). 
Aussi, les résultats obtenus montrent que les femelles de la souche Nyakabera ont atteint la première maturité sexuelle $\left(\mathrm{L}_{50}\right)$ à 10,6 cm de longueur totale contre 13,5 cm pour la souche Lac Kivu. Ces valeurs sont inférieures à celles observées par Akonkwa et al. (2016) pour O. niloticus du lac Kivu ( $\mathrm{L}_{50}$ de $15,8 \mathrm{~cm}$ Lt pour les femelles) et à celles notées (variant de $14 \mathrm{~cm}$ à $31,81 \mathrm{~cm}$ ) dans plusieurs études (Payne et Collinson, 1983 ; Peña-Mendoza et al., 2005 ; Njiru et al., 2006 ; Degefu et al., 2012 ; Tirunesh, 2015) dans divers milieux naturels. Par contre, des tailles à la première maturité sexuelle similaires $(10,1 \mathrm{~cm}$ à $11,3 \mathrm{~cm})$, pour diverses souches d'O. niloticus, ont été rapportées dans d'autres contextes d'élevage (Dhraief et al., 2010 ; Said, 2017). Selon Lowe-McConnell (1982), dans des conditions d'élevage les dimensions réduites du milieu amènent les tilapias vers une stratégie démographique caractérisée notamment par une maturité sexuelle précoce, correspondant à des tailles de plus en plus réduites.

La précocité de la maturité sexuelle des femelles de la souche Nyakabera $\left(\mathrm{L}_{50}\right.$ de $10,6 \mathrm{~cm} \mathrm{Lt}$ ) comparativement à la souche Lac Kivu $(13,5 \mathrm{~cm} \mathrm{Lt})$, toutes deux soumises aux mêmes conditions zootechniques, serait un signe d'un meilleur succès reproductif de cette souche expliqué par sa relative plus longue expérience en milieu piscicole. Contrairement à la souche du Lac Kivu, qui n'avait qu'à peine 3 ans d'acclimatation au moment de l'expérimentation, la souche Nyakabera compte une trentaine d'années de domestication depuis que son ancêtre issu du mélange entre deux souches d'O. niloticus (égyptienne et ivoirienne) est arrivé au Rwanda, en provenance des USA. En effet, d'après Osure et Phelps (2006), la domestication dans un ensemble spécifique de conditions favorise le succès reproducteur des souches ayant la plus grande histoire dans de telles conditions.

Le RGS est un aspect important de la biologie de reproduction des poissons dont une bonne compréhension améliore l'interprétation des modèles de reproduction des populations de poissons (Mahboob et Sheri, 2002), ainsi que la détermination des saisons de reproduction pour les espèces de poissons (Weng et $a$ l., 2005). En effet, chez les poissons le RGS est un indicateur de l'état des activités de reproduction ; il est corrélé au développement des gonades consécutif à une mobilisation de l'énergie en faveur de la production du sperme et des œufs (Tadesse, 1997). Les résultats du RGS obtenus montrent que les valeurs maximales sont de 5,03\% et 6,76\%, respectivement chez les femelles du Lac Kivu et de Nyakabera. Ces valeurs sont supérieures aux maxima de 3,6\% à 4,8\% rapportés par Babiker et Ibrahim (1979) et Srisakultiew et Wee (1988). Elles sont par contre proches de ceux de 6-7\% enregistrés par Melard (1986), Macintosh et al. (1988) et Shoko et al. (2015) pour les femelles de la même espèce en conditions de pisciculture.

Par rapport aux valeurs moyennes du RGS, les souches Lac Kivu et Nyakabera diffèrent significativement ( $\mathrm{p}$ $=0,005$ ), avec respectivement $2,50 \pm 0,09 \%$ et 3,04 $\pm 0,12 \%$. A la différence de RGS entre ces deux souches correspond une différence très significative $(\mathrm{p}<0,001)$ par rapport au facteur de condition $\mathrm{K}$, mais dans le sens contraire. La souche Lac Kivu avec un RGS moyen de 2,50 $\pm 0,09 \%$ se caractérise par un K moyen de $1,70 \pm 0,03$ pendant que la souche Nyakabera a un RGS moyen de 3,04 $\pm 0,12 \%$ contre un $\mathrm{K}$ moyen de 1,61 $\pm 0,02$ (Tableau 2). Une telle relation est en accord avec les observations faites sur $O$. niloticus aux lacs Tana, Ziway, Awasa et Turkana en Ethiopie, respectivement par Tadesse (1997), Tadesse (1988), Demeke (1994) et Stewart (1988), selon lesquelles aux pics de reproduction (valeurs les plus élevées du RGS) coïncident les valeurs les plus basses de K. Ces auteurs expliquent cette relation par le fait qu'une activité de reproduction accrue du poisson provoque une alimentation irrégulière et affecte par la suite l'état corporel.

Dans le cadre de l'approche comparative des deux souches évaluées ici, les différences des valeurs relatives observées pour le RGS et le facteur K seraient en lien avec celle notée pour la taille de première maturité sexuelle $\left(\mathrm{L}_{50}\right)$. La souche Nyakabera qui a été trouvée avec une petite taille de maturité sexuelle est celle-là même qui a un RGS plus élevé avec une condition relativement moins bonne. Plusieurs chercheurs ont noté que chez les poissons la $\mathrm{L}_{50}$ est liée à la condition, les poissons en mauvaise condition ayant tendance à se reproduire à des tailles plus petites que ceux qui sont en bon état corporel (Degefu et al., 2012). Les différences constatées entre les deux souches par rapport au RGS et au facteur de condition dénotent donc une différence dans la stratégie reproductive face aux conditions similaires d'élevage aménagées, avec un meilleur succès pour la souche Nyakabera. 
L'évaluation de la fécondité est l'une des exigences fondamentales pour l'étude de la biologie et de la dynamique des populations de poissons (Shalloof et Salama, 2008 ; Kingdom et Allison, 2011). Le nombre d'œufs produits par les poissons à un moment donné est un trait biologique essentiel qui attire beaucoup d'attention lorsqu'on considère les populations de poissons pour l'aquaculture. La prolifération des juvéniles avec une concurrence conséquente pour les ressources vitales peut se produire dans les structures d'élevage si les stocks de géniteurs pondent fréquemment un grand nombre d'œufs associé à un taux élevé de succès d'éclosion et une prédation négligeable (Arizi et al., 2014). Certains auteurs ont observé des différences marquées de fécondité entre les espèces qui reflètent souvent des stratégies de reproduction différentes (Murua et Saborido-Rey, 2003). Esmaeili et al. (2009) ont attribué ces différences aux effets de l'âge, de la taille des œufs et des facteurs génétiques.

Le nombre total d'ovocytes mûrs dans les ovaires (fécondité absolue) varie, pour la souche du lac Kivu, entre 279-1570 ovocytes, correspondant à une longueur totale du poisson de 14,6-22,2 cm et un diamètre ovocytaire de 0,89-2,15 mm. Pour la souche Nyakabera, la fécondité absolue varie entre 216-1504 ovocytes correspondant à une gamme de longueur totale de 11,6-18,1 cm et un diamètre ovocytaire de 11,6-18,1 mm.

Malgré la gamme réduite des tailles dans les échantillons de deux souches étudiées $(14,6-22,2 \mathrm{~cm}$ et 11,6$18,1 \mathrm{~cm}$ ), il apparaît que la fécondité absolue augmente avec la taille (voir Figure 3) comme indiqué par d'autres auteurs (Babiker et Ibrahim, 1979; Payne et Collinson, 1983; Smitherman et al., 1988 ; Bakhoum, 2002 ; Peña-Mendoza et al., 2005 ; Dhraief et al., 2010). Ainsi, la souche Lac Kivu représentée par des spécimens plus grands se caractérise par une fécondité absolue moyenne $(675 \pm 39,8)$ plus élevée que la souche Nyakabera $(508 \pm 31,9)$ dont les spécimens sont de plus petite taille. Ce constat remet en cause la signification statistique de la différence constatée entre les deux souches par rapport à ce paramètre (voir Tableau 3). La différence statistique dégagée $(p<0,001)$ entre les deux souches quant à la fécondité absolue serait réelle si on avait des gammes de poids individuels comparables dans les deux groupes. Ce qui n'est pas le cas. Par contre la fécondité relative, dont le calcul minimise l'effet taille, permet de comparer valablement les deux souches. Avec une moyenne ( \pm erreur standard) de 12,22 $\pm 0,33$ ovocytes/g de femelle, la souche Nyakabera s'est montrée plus performante que la souche lac Kivu ayant 7,70 \pm 0,38 ovocytes/g de femelle. En d'autres termes, à poids égal (et donc à taille égale) une femelle de la souche Nyakabera produit plus d'ovocytes qu'une femelle de la souche Lac Kivu. Ce résultat (différence entre souches) est en accord avec les observations des études antérieures qui indiquent d'une part que la production des œufs chez $O$. niloticus est principalement liée à la souche de la femelle (Smitherman et al., 1988) et d'autre part que la fécondité est apparemment influencée par des facteurs génétiques, ainsi que par les conditions environnementales, en particulier celles qui influencent l'état nutritionnel du poisson (Tsadik et Bart, 2007). Witthames et al. (1995) ajoutent qu'au sein d'une espèce donnée, la fécondité peut varier en fonction des différences d'adaptations à des environnements précis (reflet de la différence dans la stratégie de reproduction évoquée plus haut).

Pour les deux souches, la fécondité absolue est directement corrélée à la longueur totale, au poids total et au poids des ovaires. Par contre, aucune corrélation n'a été observée entre la fécondité absolue et le diamètre ovocytaire. Ce résultat concorde bien avec ceux trouvés par Shoko et al. (2015) pour O. niloticus élevé en étang en monoculture et en polyculture avec Clarias gariepinus et par Shalloof et Salama (2008), pour $O$. niloticus dans un lac égyptien. L'absence de corrélation entre la fécondité et le diamètre ovocytaire s'explique, d'après Shoko et al. (2015), par le fait que chez les poissons la taille des ovocytes mûrs ne varie pas beaucoup avec la taille ou l'âge du poisson.

L'observation concernant le succès reproductif de la souche Nyakabera par rapport à la souche Lac Kivu est en accord avec celle faite par Osure et Phelps (2006). Ces auteurs avaient trouvé que les souches «Egypte » et «Côte d'Ivoire» du tilapia du Nil, domestiquées dans des conditions similaires pour un certain nombre d'années, avaient eu plus de succès reproductif que les souches «Lac Victoria » et «Sagana » plus récemment introduites en pisciculture. La différence constatée dans le comportement reproductif des 
souches Lac Kivu et Nyakabera est expliquée par une différence dans la réponse adaptative aux conditions de l'environnement piscicole. Cependant, un soubassement génétique de cette différence ne serait pas à exclure. Uraiwan (1988) confirme qu'une relation existe, par exemple, entre le taux de croissance, l'âge et la taille à la maturité sexuelle chez le tilapia du Nil. Les deux souches comparées sont en effet différenciées sur le plan morphologique (Nihoreye et al., soumis), ce qui peut présager une différenciation génétique. Aussi, elles se caractérisent par des origines lointaines bien distinctes associées à une absence de flux génétique. A ce propos, Duponchelle et al. (2000) notent que 25 ans (soit 50 générations) de séparation entre des populations (d'O. niloticus) peuvent être suffisants pour induire des différences génétiques dans les traits reproductifs.

\section{Conclusion}

Les deux souches d'O. niloticus comparées dans cette étude ont présenté des différences pour les traits reproductifs tels que la taille de maturité sexuelle, le rapport gonado-somatique, le facteur de condition et la fécondité. Ces différences seraient en lien avec l'origine et l'histoire de domestication toutes aussi différentes de ces deux souches. La souche Nyakabera, descendant des souches égyptienne et ivoirienne et ayant évolué depuis plusieurs années dans les conditions d'élevage, s'est montrée plus performante sur le plan reproductif (RGS et fécondité relative plus élevés, maturité sexuelle précoce) que la souche naturelle du lac Kivu, fraîchement introduite en pisciculture.

Il serait intéressant de vérifier à l'avenir si les différences observées au niveau des performances de reproduction, et mises en lien avec la différenciation morphologique de deux souches comparées, sont aussi corrélées à une différenciation génétique.

\section{Remerciements}

Les auteurs remercient les membres du staff technique du Service National d'Aquaculture (SENAQUA), Coordination provinciale du Sud-Kivu, pour leur sens de coopération pendant la réalisation du volet expérimental de cette étude. Ils pensent particulièrement à Monsieur André Bandibabusire qui n'a ménagé aucun effort pour assurer la surveillance des étangs et les tâches de routine pendant la période d'élevage.

\section{Références}

[1] Akonkwa, B., Ahouansou-Montcho, S., Nshombo, M. et Lalèyè, P. 2016. Quelques aspects de la reproduction des poissons du lac Kivu. Afrique SCIENCES, 12(4): 240-255.

[2] Albaret, J.J. 1982. Reproduction et fécondité des poissons d'eau douce de Côte d'Ivoire. Revue d'Hydrobiologie tropicale, 15 (4): 347-371: http://horizon.documentation.ird.fr/exldoc/pleins_textes/cahiers/hydrob-trop/03550.pdf

[3] Almeida, D.B., da Costa, M.A.P, Bassini, L.N., Calabuig, C.I.P, Moreira, C.G.A, Rodrigues, M.D.N, Pérez, H.J., Tavares, R.A, Varela Jr, A.S. and Moreira, H.L.M. 2013. Reproductive performance in female strains of Nile tilapia, Oreochromis niloticus. Aquaculture International, 21: 1291-1300: https://link.springer.com/article/10.1007/s10499-013-9630-0

[5] Arizi E.K., Obodai, E.A and Aggrey-Fynn, J. 2014. Reproductive Biology of Sarotherodon melanotheron in the Dominli Lagoon, Ghana. International Journal of Ecology and Environmental Sciences, 40 (4): 245-253: https://fishcomghana.com/wp-content/uploads/2017/04/Reproductive-Biology-of-Sarotherodonmelanotheron-in-the-Dominli-Lagoon-Ghana.pdf

[6] Babiker, M.M. and Ibrahim, H. 1979. Studies on the biology of reproduction in the cichlid Tilapia nilotica (L.): gonadal maturation and fecundity. Journal of Fish Biology, 14: 437-448.

[7] Bagenal, T.B. 1978. Methods for assessment of fish production in freshwaters. Blackwell Scientific Publications, Oxford, $365 \mathrm{p}$.

[8] Bakhoum, S.A. 2002. Comparative reproductive biology of the Nile tilapia Oreochromis niloticus (L.), blue tilapia, Oreochromis aureus (Steind.) and their hybrids in Lake Edku, Egypt. Journal of 
Aquatic Biology and Fisheries, Vol. $\quad 6, \quad \mathrm{~N}^{\circ} 3$ : $121-142$ : http://ejabf.journals.ekb.eg/article_1753_c677a44cf0888dcd619f590a456fc67e.pdf

[9] Berté, S., Kouamélan, E.P, Ouattara, N.I, Koné, T., Goore, B.G, N’Douba, V. et Kouassi, N.J. 2008. Cycle de reproduction et fécondité de Distichodus rostratus (Characiformes, Distichodontidae) dans un bassin ouest africain (fleuve Bandama, Côte d'Ivoire). TROPICULTURA, 26(2): 104-107: https://www.researchgate.net/.../45266295 Cycle de reproduction et fecondite de Dis...

[10] Bolivar, R.B., Eknath, A.E., Bolivar, H.L. and Abella, T.A. 1993. Growth and reproduction of individually tagged Nile tilapia (Oreochromis niloticus) of different strains. Aquaculture, 111: 159169.

[11] Degefu, F., Tesfaye, G. and Tefera, F. 2012. Study on the Adaptability Status and Reproductive Success of Oreochromis niloticus L. (Pisces: Cichlidae) and Carp (Cyprinus carpio L., 1758) in a Tropic Reservoir (Fincha, Ethiopia). International Journal of Aquaculture, Vol.2, $\mathrm{N}^{\circ}$ 10: 65-71: https://www.researchgate.net/.../259282867_Study_on_the_Adapta...

[13] De Graaf, G.J., Galemoni, F. and Huisman, E.A. 1999. Reproductive biology of pond reared Nile tilapia Oreochromis niloticus L. Aquaculture Research, 30: 25-33.

[14] Demeke, A. 1994. Maturity, fecundity, brood-size and sex-ratio of Tilapia (Oreochromis niloticus L) in Lake Awassa. SINET: Ethiopian Journal of Science, 17(1): 53-69.

[15] De Vlaming, V., Grossman, G.D. and Chapman, F. 1982. On the use of the gonado-somatic index. Comp. Biochem. Physiol., 73: 31-39.

[16] Dhraief, M., Azaza, M.S. et Kraiem, M. 2010. Etude de la reproduction du Tilapia du Nil Oreochromis niloticus (L.) en captivité dans les eaux géothermales du sud tunisien. Bulletin de l'Institut National des Sciences et Technologies de la Mer de Salammbô, 37: 89-96: http://www.instm.agrinet.tn/images/Bulltin/Bull.2010/11.pdf

[17] Dunham, R.A. and Smitherman, R.O. 1984. Ancestry and breeding of catfish in the United States. Circular 273, Alabama Agricultural Experiment Station Auburn University, AL, 100 p.

[18] Duponchelle, F., Pouyaud, L. and Legendre, M. 1998. Evidence of environmental effects on reproductive characteristics of Nile tilapia (Oreochromis niloticus) populations from man-made lakes of Ivory Coast. Aquatic Living Resources, 11(3): 137-144: http://horizon.documentation.ird.fr/exldoc/pleins textes/pleins textes 7/b fdi 51-52/010014887.pdf

[19] Duponchelle, F., Cecchi, P.H., Corbin, D., Nuñez, J. and Legendre, M. 2000. Variations in fecundity and egg size of female Nile tilapia, Oreochromis niloticus, from man-made lakes of Côte d'ivoire. Environmental Biology of Fishes, 57:155-170.

[20] Eguia, M.R.R. 1996. Reproductive performance of four red tilapia strains in different seed production systems. The Israeli Journal of Aquaculture-Bamidge, 48: 10-18.

[21] Engle, C.R. and Neira, I. 2005. Tilapia farm business management and economics: a training manual. Aquaculture CRSP, Oregon State University, Corvallis, 41 p.

[22] Esmaeili, H.R., Ganjali, Z. and Monsefi, M. 2009. Reproductive biology of the endemic Iranian cichlid, Iranocichla hormuzensis Coad, 1982 from Mehran River, southern Iran. Environmental Biology of Fishes, 84: 141-145:

https://www.researchgate.net/.../248774339_Reproductive_biology...

[24] Fermon, Y. 1996. Les Haplochromis spp (Teleostei, Cichlidae) des zones rocheuses du Mwanza Gulf, Lac Victoria, Tanzanie : structure des communautés et écomorphologie. TDM. ORSTOM. Paris. 270 p.

[25] Gourène, G. and Teugels, G.G. 1993. Différenciation morphologique de souches des tilapias Oreochromis niloticus et $O$. aureus (Teleostei, Cichlidae) utilisées en pisciculture. Cybium, 17: 343 355: https://www.ajol.info/index.php/scinat/article/viewFile/42148/9276

[26] Hammer, Ø., Harper, D.A.T and Ryan, P.D. 2001. PAST: Paleontological Statistics Software Package for Education and Data Analysis. Palaeontologia Electronica, 4(1): 9 p.

[27] Hunter, J.R., Macewicz, B.J., Lo, N.C. and Kimbrell, C.A. 1992. Fecundity, spawning and maturity of female Dover sole Microstomus pacificus, with an evaluation of assumptions and precision. Fishery Bulletin US, 90: 101-128: https://swfsc.noaa.gov/publications/CR/1992/9246.PDF 
[28] Kamel, E.A.R. 2011. Evaluation of Reproductive Performance of Tilapia Strains and Some of Their Crosses. Journal of the Arabian aquaculture society, Vol. 6, No 2, 119-136: http://www.arabaqs.org/journal/vol_6/2/Text11-10.pdf

[29] Kincaid, H.L. 1981. Trout strain registry. FWS/NFC-L/81-1. U.S. Fish and Wildl. Serv. KearNeysville, WV, 118 p.

[30] Kingdom, T. and Allison, M.E. 2011. The fecundity, gonadosomatic and hepatosomatic indices of Pellonula leonensis in the lower Nun river, Niger delta, Nigeria. Current Research Journal of Biological Sciences, 3: 175-179:

https://www.researchgate.net/.../286930871 The Fecundity Gona...

[32] Legendre, M. and Ecoutin, J.M. 1989. Suitability of brackish water tilapia species from the Ivory Coast for lagoon aquaculture. I-Reproduction. Aquatic Living Resources, 2: 71-79.

[33] Legendre, M. et Ecoutin, J.M. 1996. Aspects de la stratégie de reproduction de Sarotherodon melanotheron, comparaison entre une population naturelle (lagune Ebrié, Côte d'Ivoire) et différentes populations d'élevage, p. 360-374. In R.S.V. Pullin, J. Lazard, M. Legendre, J.B. Amon Kothias et D. Pauly (éds.) Le Troisième Symposium International sur le Tilapia en Aquaculture. ICLARM Conf. Proc. 41, 630 p.

[34] Little, D.C. 1989. An evaluation of strategies for production of Nile tilapia (Oreochromis niloticus L.) fry suitable for hormonal treatment. PhD Dissertation, Institute of Aquaculture, University of Stirling, Scotland, UK. 376p.

[35] Lowe-McConnell, R.H. 1982. Tilapia in fish communities. In: Pullin, R.S.V. and LoweMcConnell, R.H. (Eds.), The Biology and Culture of Tilapias. ICLARM Conference Proceeding 7, International Center for Living Aquatic Resources Management, Manila Philippines: 83-113.

[36] Macaranas, J.M., Agustin, L.Q., Ablan, M.C.A., Pante, M.J.R., Eknath, A.A. and Pullin, R.S.V. 1995. Genetic improvement of farmed tilapias: biochemical characterization of strain differences in Nile tilapia. Aquaculture International, 3: 43-54.

[37] Macintosh, D.J., Singh, T.B., Little, D.C. and Edwards, P. 1988. Growth and sexual development of 17- $\alpha$-methyltestosterone and progresterone treated Nile tilapia (Oreochromis niloticus) reared in earthen ponds. In: The Second International Symposium on Tilapia in Aquaculture. ICLARM Conference Proceedings 15 (ed. by R.S.V. Pullin, T. Bhukaswan, T. Tonguthai \& J.L. Maclean), pp. 457- 463. ICLARM, Manila.

[38] Mair, G.C., Lakapunrat, S., Jere, W.L. and Bart, A. 2004. Comparisons of reproductive parameters among improved strains of Nile Tilapia Oreochromis niloticus L. Proceeding of the Sixth International Symposium on Tilapia in Aquaculture, Manila, Philippines (ed. by R. Bolivar, G. Mair \& K. Fitzsimmons), pp.142-156.

[39] Mahboob, S. and Sheri, A.N. 2002. Relationship among gonad weight, liver weight and body weight of major, common and some Chinese carps under composite culture system with special reference to pond fertilization. Asian-Australian Journal of Animal Science, 15: 740-744: https://www.researchgate.net/.../256426542_Relationships_among_...

[40] Melard, C. 1986. Les bases biologiques de l'élevage intensif du Tilapia du Nil, Recherches sur la biologie d'Oreochromis (Tilapia) niloticus L. (Pisces, Cichlidae) en élevage expérimental : reproduction, croissance, bioénergétique. Cahiers d'Ethologie Appliquée, Vol. 5, № 3, pp. 1-224.

[41] Murua, H. and Saborido-Rey, F. 2003. Female reproductive strategies of marine fish and their classification in the North Atlantic. Journal of Northwestern Atlantic Fisheries Sciences, Vol. 33: 23-31: http://digital.csic.es/bitstream/10261/26868/1/murua.pdf

[42] Nihoreye, F., Muzumani, R. et Nshombo, M. 2009. Résultats préliminaires sur la différenciation morphologique des deux souches du tilapia du Nil Oreochromis niloticus (Teleostei, Cichlidae) utilisées en pisciculture à l'Est de la R.D. Congo. Cahier du CERUKI, Nouvelle série, 39: 192-200.

[43] Nihoreye, J.F., Masilya, P.M., Isumbisho, P.M. et Okitayela, F.O. (sous presse). Différenciation morphologique entre deux souches naturelles et une souche cultivée du tilapia du Nil Oreochromis niloticus (Linnaeus, 1758) au Sud-Kivu, RD Congo. 
[44] Njiru, M., Ojuok, J.E., Okeyo-Owuor,, J.B., Muchiri, M., Ntiba, M.J. and Cow, I.G. 2006. Some biological aspects and life history strategies of Nile tilapia Oreochromis niloticus (L.) in Lake Victoria, Kenya. African Journal of Ecology, 44: 30-37.

[45] Osure, G.O. and Phelps, R.P. 2006. Evaluation of reproductive performance and early growth of four strains of Nile tilapia (Oreochromis niloticus, L.) with different histories of domestication. Aquaculture, 253(1-4): 485-494.

[46] Pauly, D. 1993. Fishbyte section. Editorial. Naga. ICLARM contribution. 16, 26.

[47] Payne, A.I. and Collinson, R.I. 1983. A comparison of the biological characteristics of Sarotherodon niloticus (L.) With those of S. aureus (Steindachner) and other Tilapia of the delta and lower Nile. Aquaculture, 30: 335-351.

[48] Peña-Mendoza, B., Gómez-Márquez, J.L., Salgado-Ugarte, I.H. and Ramírez-Noguera, D. 2005. Reproductive biology of Oreochromis niloticus (Perciformes: Cichlidae) at Emiliano Zapata dam, Morelos, Mexico. International Journal of Tropical Biology, Vol. 53(3-4): 515-522: https://revistas.ucr.ac.cr/index.php/rbt/article/viewFile/14666/13910

[49] Rana, K.J. and Macintosh, D.J. 1988. A comparison of the quality of hatchery-reared Oreochromis niloticus and Oreochromis mossambicus fry. In: Tilapia in Aquaculture. The Second International Symposium On Tilapia in Aquaculture, Bangkok, Thailand, ICLARM Conference Proceedings, Vol.15 (ed. by R.S.V. Pullin,T. Bhukaswan,T. Tonguthai \& J. L. Maclean), pp.497-502. ICLARM, Manila, Philippines.

[50] Said, M.M. 2017. Evaluation of early maturity aspects of two egyptian Nile tilapia (Oreochromis niloticus) strains: Abbassa improved and Manzala commercial within a diallel crossing. Egyptian Journal of Animal Production, 54(1): 67-75:

https://www.researchgate.net/.../313794561_Evaluation_of_early_...

[52] [47] Shalloof, K.A.S. and Salama, H.M.M. 2008. Investigations on Some Aspects of Reproductive Biology in Oreochromis niloticus (Linnaeus, 1757) Inhabited Abu-zabal Lake, Egypt. Global Veterinaria, 2(6): 351-359: http://citeseerx.ist.psu.edu/viewdoc/download?doi=10.1.1.564.2137\&rep=rep1\&type=pdf

[53] Shoko, A. P., Limbu, S.M., Mrosso, H.D.J. and Mgaya, Y.D. 2015. Reproductive biology of female Nile tilapia Oreochromis niloticus (Linnaeus) reared in monoculture and polyculture with African sharptooth catfish Clarias gariepinus (Burchell). SpringerPlus, 4: 25:

https://www.ncbi.nlm.nih.gov/pubmed/26090322

[55] Smitherman, R.O., Khater, A.A., Cassell, N.I. and Dunham, R.A. 1988. Reproductive performance of three strains of Oreochromis niloticus. Aquaculture, 70: 29-37:

https://eurekamag.com/pdf/001/001673323.pdf

[56] Snoeks, J., De Vos, L. and Thys van den Audenaerde, D.F.E. 1997. The ichthyogeography of Lake Kivu. South African Journal of Science, Vol. 93, 579-584.

[57] Srisakultiew, P. and Wee, K.L. 1988. Synchronous spawning of Nile Tilapia through hypophysation and temperature manipulation. In: The Second International Symposium on Tilapia in Aquaculture. ICLARM Conference Proceedings 15 (ed. by R. S. V. Pullin, T. Bhukaswan, K. Tonguthai \& J. L. Maclean), pp. 275-284. ICLARM, Manila.

[58] Stearns, S.C. 1983. The genetic basis of differences in life history traits among six populations of mosquito fish (Gambusia affinis) that shared ancestors in 1905. Evolution, 37: 618-627.

[59] Stewart, K.M. 1988. Changes in condition and maturation of the Oreochromis niloticus L. population of Ferguson's Gulf, Lake Turkana, Kenya. Journal of Fish Biology, 33: 181-188.

[60] esse, Z. 1988. Studies on some aspects of the biology of Oreochromis niloticus L. (Pisces: Cichlidae) in Lake Ziway, Ethiopia, MSc Thesis, Addis Ababa University, Addis Ababa, 78 p.Tadesse, Z. 1997. Breeding season, fecundity, length-weight relationship and condition factor of Oreochromis niloticus

[61] L. (Pisces: Cichlidae) in Lake Tana, Ethiopia, SINET: Ethiopian Journal of Science, 20(1): 31-47:

[62] Tirunesh, S.G. 2015. Conceptive science of Oreochromis niloticus in Lake Beseka, Ethiopia. International Journal of Cell and Animal Biology, Vol. 2(3), 84-87: https://docplayer.net/35540005-Conceptive-science-oforeochromis-niloticus-in-lake-beseka-ethiopia.html 
[63] Tsadik, G.G. and Bart, A.N. 2007. Characterization and comparison of variations in reproductive performance of Chitralada strain Nile tilapia, Oreochromis niloticus (L.). Aquaculture Research, 38: 1066-1073.

[64] Uraiwan, S. 1988. Direct and indirect responses to selection for age at first maturation of Oreochromis niloticus, in: Pullin, R.S.V., Bhukaswan, T., Tonguthai, K.,Maclean, J.L. (Eds.), The Second International Symposium on Tilapia in Aquaculture. Department of Fisheries, Thailand and International Center for Living Aquatic Resources Management, Bangkok, pp. 295-300.

[65] Vreven, E.J., Adépo-Gourène, B., Agnèse, J.F. and Teugels, G.G. 1998. Morphometric and allozyme variation in natural populations and cultured strains of the Nile tilapia Oreochromis niloticus (Teleostei, Cichlidae). Belgian Journal of Zoology, 128(1): 23-34: http://horizon.documentation.ird.fr/exl-doc/pleins_textes/pleins_textes_7/b_fdi_51-52/010015998.pdf

[66] Weng, J.S., Liu, K.M., Lee, S.C. and Tsai, W.S. 2005. Reproductive biology of the blue sprat Spratelloides gracilis in the waters around Penghu, Central Taiwan Strait. Zoological Studies, 44: 475-486: http://zoolstud.sinica.edu.tw/Journals/44.4/475.pdf

[67] Witthames, E.R., Greer-Walker, M., Dinis, M.T. and Whiting, C.L. 1995. The geographical variation in the potential annual fecundity of Dover sole Solea solea (L.) from European shelf waters during 1991. Netherlands Journal of Sea Research, 34(1-3): 45-58. http://www.fishbase.org/physiology/strainlist.php?ID=2\&SortBy=StrainCode_ (consulté le 15/06/2018).

\section{Résumé}

Cette étude a été conduite pour évaluer les performances de reproduction des femelles d'Oreochromis niloticus provenant d'une souche d'origine lointaine et longuement exploitée en pisciculture (souche Nyakabera) et d'une souche locale récemment prélevée du milieu naturel (souche Lac Kivu). L'expérimentation a été réalisée en triplicata avec des alevins de 4,9 à 6,3 g placés dans des étangs en terre pour une durée de 6 mois, à la station piscicole de Nyakabera au Sud-Kivu, République Démocratique du Congo. La comparaison de deux souches a porté sur la taille de première maturité sexuelle, le rapport gonado-somatique, la fécondité, le diamètre des ovules ainsi que le facteur de condition. Les résultats ont montré que dans les conditions d'élevage similaires la souche Nyakabera atteint la maturité sexuelle plus précocement et se caractérise par un rapport gonado-somatique et une fécondité relative plus élevés mais une condition moins bonne par rapport à la souche Lac Kivu. Ces différences constatées, qui dénotent un meilleur succès reproductif de la souche Nyakabera, seraient en lien avec l'origine et l'histoire de domestication toutes aussi différentes de ces deux souches.

Mots clés: Fécondité, Lac Kivu, Pisciculture, Rapport gonado-somatique, Taille de première maturité sexuelle 